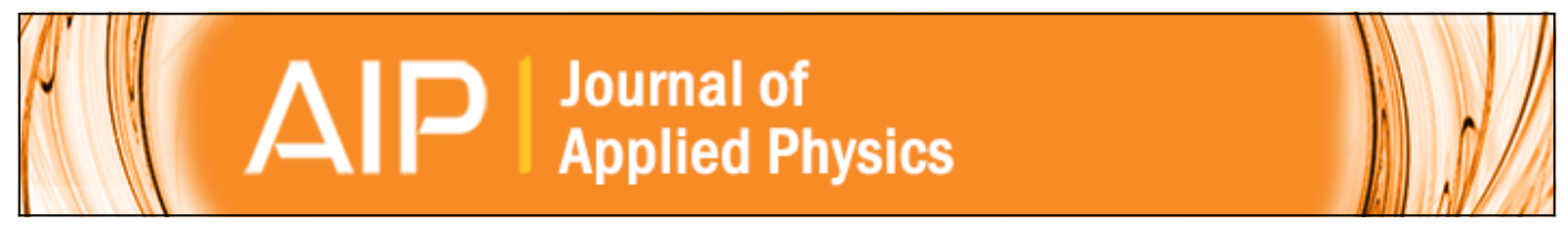

\title{
Doping of polyaniline by corona discharge
}

Aldo E. Job, José A. Giacometti, Paulo S. P. Herrmann Jr., and Luiz H. C. Mattoso

Citation: Journal of Applied Physics 87, 3878 (2000); doi: 10.1063/1.372428

View online: http://dx.doi.org/10.1063/1.372428

View Table of Contents: http://scitation.aip.org/content/aip/journal/jap/87/8?ver=pdfcov

Published by the AIP Publishing

\section{AlP}

\section{Create a profile. \\ Sign up today!}




\title{
Doping of polyaniline by corona discharge
}

\author{
Aldo E. Job \\ Faculdade de Ciências e Tecnologia, Universidade Estadual Paulista, C.P. 379 Presidente Prudente, \\ 19060-900, SP, Brazil \\ José A. Giacomettia) \\ Instituto de Física de São Carlos, Universidade de São Paulo, C.P. 369 São Carlos, 13560-970, SP, Brazil \\ Paulo S. P. Herrmann, Jr. and Luiz H. C. Mattoso \\ Embrapa Instrumentação Agropecuária, CNPDIA/EMBRAPA, C.P. 741, São Carlos, 13560-970, SP, Brazil
}

(Received 8 September 1999; accepted for publication 6 January 2000)

In the present article it is shown that a corona discharge can be employed to dope thin films of polyaniline (PANI) coated on poly(ethylene terephthalate) films, allowing the electrical conductivity to be tuned within the range $10^{-10}$ to $0.3 \mathrm{~S} \mathrm{~cm}^{-1}$. A study of the effect of different corona conditions, namely corona treatment for positive and negative polarities, air humidity, treatment time, corona current, and the geometry of the corona triode, on the electrical conductivity of the polyaniline is presented. The results indicate that the corona discharge leads to protonic doping of polyaniline similar to that which occurs in conventional protonic acid solution doping. Atomic force microscopic analysis shows that, as the PANI is exposed to the corona discharge, its globular morphology is disrupted leading to the appearance of droplet-like features and a significant decrease in the average height and surface roughness. Doping by corona discharge presents several advantages over the conventional solution method namely that it is a dry process which does not require use of chemicals reagents, and which is both rapid and avoids dopant migration. The latter can be important for applications of PANI in microelectronic devices. (C) 2000 American Institute of Physics. [S0021-8979(00)01608-X]

\section{INTRODUCTION}

Polyaniline (PANI) in its nonconducting form was discovered more than 100 years ago, but it only became an interesting conducting polymer after the advent of doping by protonic acids, owing to its importance for the study of fundamental aspects of charge transport as well as its great potential for application in the construction of devices. PANI also offers advantages such as its low preparation cost, ease of processibility, and environmental stability. An enormous amount of research ${ }^{1-6}$ has been done on doping processes in order to enhance the electrical conductivity of the polymer. In this context, several doping approaches have been proposed whose suitability depends on the final application. For instance, using aqueous acidic solutions in chemical or electrochemical ${ }^{1}$ doping processes pose limitations if the resultant polyanilines are to be used for a number of technological applications, ${ }^{2}$ particularly in the microelectronic industry. ${ }^{3,4}$ Alternative methods involving doping of polyaniline with high-energy radiation such as e-beam, ${ }^{4} \mathrm{x}$ rays ${ }^{5}$ and $\gamma$ radiation, ${ }^{6}$ on the other hand, have disadvantages which include their high cost, low efficiency, and the possible resultant radiation damage.

An approach that does not suffer of any of these drawbacks is doping via corona discharge, which we have recently applied, ${ }^{7-9}$ to polyaniline coated on top of poly(ethylene terephthalate) (PET) films. In the corona method-which has been extensively used for charging polymers, ${ }^{10}$ treating

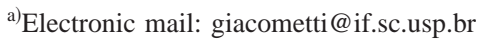

polymer surfaces, ${ }^{11,12}$ in electrophotocopying machines, ${ }^{13}$ and in electrostatic filters, ${ }^{14}$ - a high voltage is applied to an electrode such as a metallic point. ${ }^{15}$ Excited neutral species and positive or negative ions, depending on the voltage polarity, are produced in a region near the corona point. Mechanical collisions in the discharge region lead to gas movement, which is usually referred to as corona wind. Upon applying a corona discharge, neutral species are driven towards the sample surface by the corona wind while charged ions are driven by electrostatic attraction.

In this article we show detailed results on the influence of corona discharge experimental conditions on the electrical conductivity and properties of PANI deposited onto PET films, referred to as PET/PANI composites. The species responsible for the doping process are proposed in accordance with the literature. The implications of this doping method for the application of polyanilines with an antistatic function ${ }^{16}$ in electronic packaging are briefly discussed.

\section{EXPERIMENT}

PET films were supplied by Rhodia-Ster/Brazil (Terphane $\left.{ }^{\circledR}\right)$ with nominal thickness of $12 \mu \mathrm{m}$. Coating of PET films with PANI was performed by chemically synthesizing parent polyaniline in a polymerization solution in which a PET film was immersed. The synthesis was carried out during approximately $60 \mathrm{~min}$ following the conventional chemical method ${ }^{17}$ at room temperature using aniline and ammonium peroxydisulfate in a 1.0 molar $\mathrm{HCl}$ solution. The PET/ PANI composite was deprotonated in a 0.1 molar ammonium 


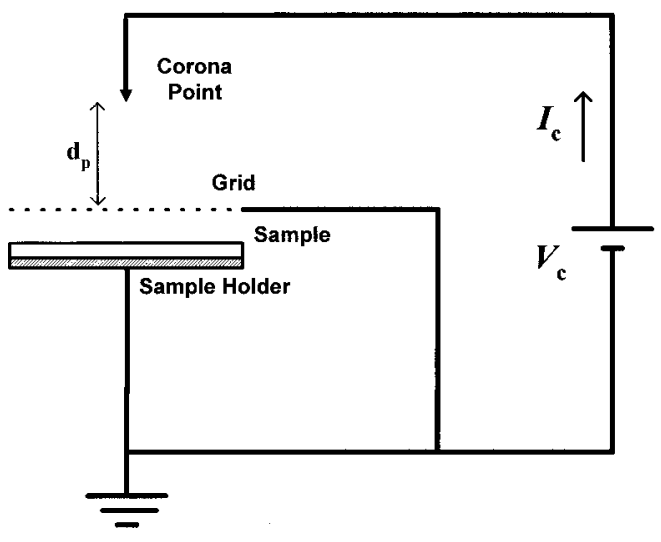

FIG. 1. Schematic representation of the corona triode used to treat the PET/PANI composite. The corona triode was mounted inside a chamber having two gas outlets and heaters allowing for the control of the relative air humidity and temperature. $l_{c}$ is the corona current, $d_{p}$ is the distance between the corona point and the metallic grid, and $V_{c}$ is the corona voltage supply.

hydroxide solution for $24 \mathrm{~h}$ and then dried under vacuum, in order to obtain PANI in the dedoped emeraldine base (EB) oxidation state. The PANI layer deposited by this method is well attached to the PET film, according to a detailed study of the composites. ${ }^{18}$

The PET/PANI composites were characterized by UVVis absorption spectroscopy using a Perkin-Elmer model Lambda-9 Spectrophotometer. Electrical conductivity of the PANI coating was measured with the four-point probe technique at room temperature. The conductivity stability was monitored at room temperature for samples treated for $70 \mathrm{~h}$ at distinct temperatures. Corona discharge was generated using a corona triode system, shown schematically in Fig. 1, consisting of a metallic point, a stainless steel grid, and a sample holder. ${ }^{10}$ The distance from the grid to the point, $d_{p}$, could be varied in the range from 1 to $5 \mathrm{~cm}$. The grid to sample distance was kept constant at $0.5 \mathrm{~cm}$. A reversible dc voltage supply (up to $\pm 20 \mathrm{kV}$ ) was connected to the tip in order to generate positive or negative corona discharge. During the treatment the corona current, $I_{c}$, was kept constant. In measurements described in Sec. III both the grid and sample holder were grounded to avoid the film being charged at very high surface potentials, thus preventing breakdown of the PET/PANI composite. Corona treatments were performed in air at room temperature $\left(\sim 25^{\circ} \mathrm{C}\right)$, unless otherwise specified. The corona setup was mounted inside a stainless steel chamber, which allowed the relative air humidity to be varied from $0 \%$ to $100 \%$, within an accuracy of $\pm 2 \%$.

Atomic force microscopy (AFM) measurements were carried out using a Topometrix Discoverer TMX 2000 instrument in the standard contact mode using $200-\mu \mathrm{m}$-long V shaped cantilevers with a nominal spring constant of 0.06 $\mathrm{N} / \mathrm{m}$ and $\mathrm{Si}_{3} \mathrm{~N}_{4}$ pyramidal tips integrated onto the underside of the cantilever end. The scan rate was $1.25 \mathrm{~Hz}$ and AFM images were collected with $300 \times 300$ data points. The surface roughness was estimated by calculating the average roughness $(\mathrm{Ra})$ and the root mean square (rms) roughness using software supplied with the Topometrix instrument. The thickness of the PANI coating was determined by partially

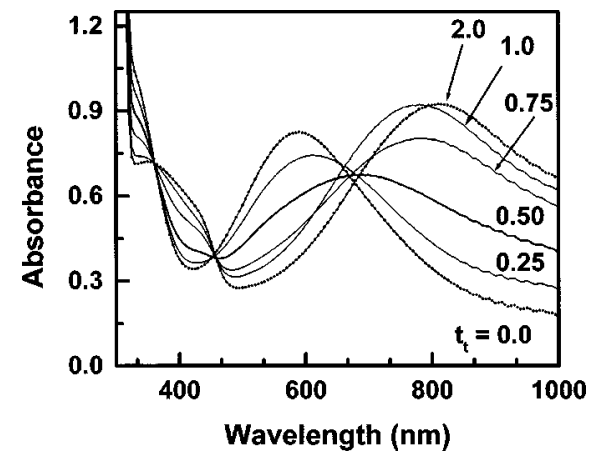

FIG. 2. UV-Vis spectra for PET/PANI composite films treated with positive corona during different periods of time, $t_{t}$. The corona current was +15 $\mu \mathrm{A}$, the relative air humidity was $60 \%$, and $d_{p}=1.3 \mathrm{~cm}$. The treatment time is indicated for each curve and $t_{t}=0$ corresponds to the PANI-EB.

removing the coating with scotch tape and measuring the height of the step formed, which was of the order of $150 \mathrm{~nm}$.

\section{RESULTS}

The UV-Vis electronic absorption spectra of polyaniline reflect its doping state. For as-prepared PET/PANI composites, in which PANI is in the dedoped EB state, Fig. 2 shows a peak with $\lambda_{\max }$ at approximately $600 \mathrm{~nm}$ (transition energy of $2.07 \mathrm{eV}$ ), assigned to an excitonic transition. Upon exposing this PET/PANI composite to a positive corona discharge this peak is shifted towards higher wavelengths owing to a decrease in the energy associated with the electronic transitions, and a shoulder appears at around $400 \mathrm{~nm}$. $\lambda_{\max }$ increases with increasing corona discharge time, reaching 850 $(1.46 \mathrm{eV})$ and $800 \mathrm{~nm}(1.55 \mathrm{eV})$ for positive and negative corona, respectively. The shift is characteristic of doping of polyaniline, similarly to that which occurs for PANI doped by other methods. ${ }^{1,4,17}$ Doping was confirmed by measuring the electrical conductivity of the PANI coating, which can be increased from $10^{-10}$ to $0.3 \mathrm{~S} \mathrm{~cm}^{-1}$ with corona treatment (Fig. 3), therefore reaching the levels achieved with conventional $\mathrm{HCl}$ doping. In addition, the conductivity remains stable for long periods of time, as will be shown later. The effect of the experimental conditions under which the corona discharge was applied on the electrical conductivity of the PANI coating is illustrated in Figs. 4-6. Doping efficiency

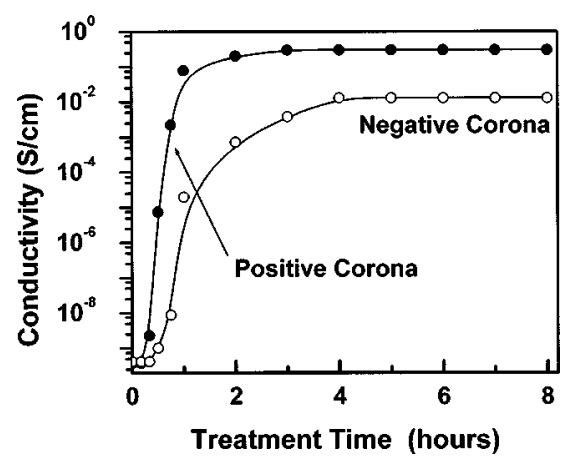

FIG. 3. Dependence of the electrical conductivity for PET/PANI composite films with positive and negative corona during different period of time. The corona current was $\pm 15 \mu \mathrm{A}$, the relative air humidity was $60 \%$ and $d_{p}$ $=1.3 \mathrm{~cm}$. 


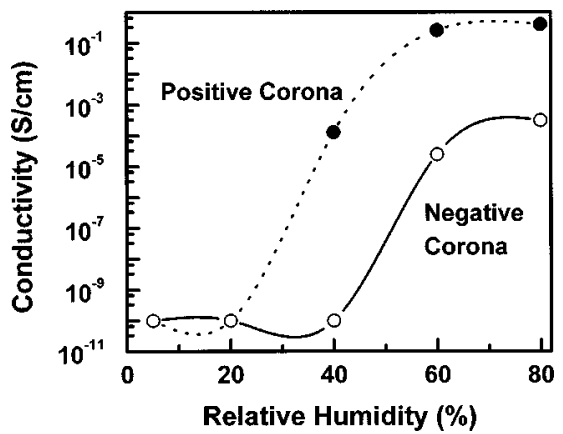

FIG. 4. Dependence of the electrical conductivity for PET/PANI composite films with positive and negative corona for different values of the relative air humidity. The corona current was $\pm 15 \mu \mathrm{A}$, the treatment time was $1 \mathrm{~h}$ and $d_{p}=1.3 \mathrm{~cm}$.

increases considerably after a given relative humidity and is higher for positive corona, as demonstrated in Fig. 4. The maximum conductivity for positive corona treated samples reached $0.3 \mathrm{~S} / \mathrm{cm}$ while that for negative corona value was limited to the order of $10^{-3} \mathrm{~S} / \mathrm{cm}$. In addition, for negative corona the environment requires a higher humidity and takes a longer time for the doping to occur than for positive corona.

The electrical conductivity of the PANI coating increases with the corona current (Fig. 5) for a fixed treatment time, as expected. Here there is a limitation in the maximum current that can be used before producing sparks between the corona point and the grid. Again the positive corona is more effective for doping the PANI coating. It is known that an increase in the corona current leads to an increase in the amount of species produced in the metallic tip by the corona discharge, and therefore the amount of doping species is expected to increase, as well. The efficiency of the treatment is influenced by corona discharge parameters such as the corona current and point-to-plane distance. As shown in Fig. 6, when the distance between the corona point and the metallic grid is increased, the electrical conductivity tends to decrease, for a given corona current. Apparently, as one increases the distance between the corona point and the sample, the number of doping species that reach the sample decreases. Figure 7 shows that upon increasing the tempera-

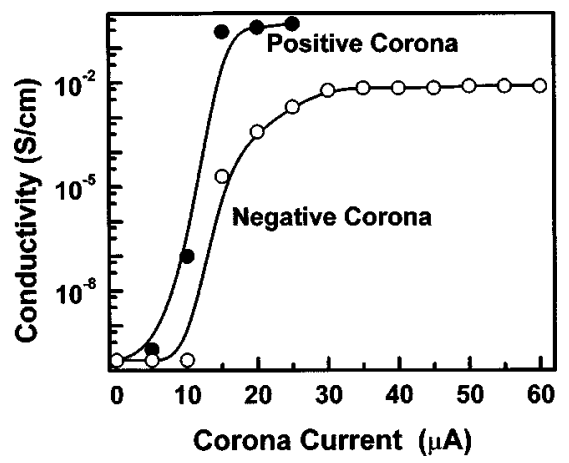

FIG. 5. Dependence of the electrical conductivity for PET/PANI composite films with positive and negative corona on the corona current. The treatment time was $1 \mathrm{~h}$ and $d_{p}=1.3 \mathrm{~cm}$. Above values of $I_{c}=-25$ and $+60 \mu \mathrm{A}$ the electric breakdown in the gap between the corona and the grid occurred.

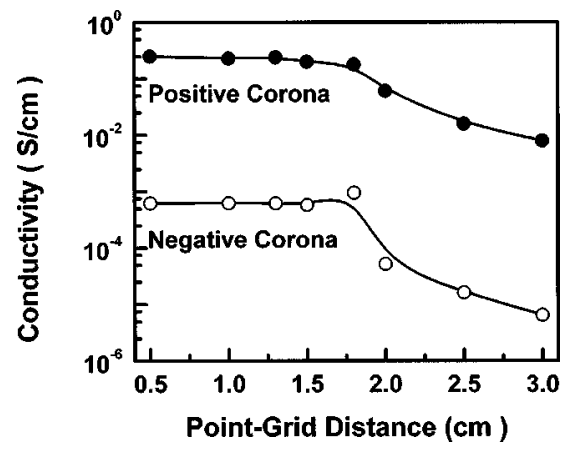

FIG. 6. Dependence of the electrical conductivity for PET/PANI composite films with the distance between the corona point and the grid, $d_{p}$, for a corona current $I_{c}= \pm 15 \mu \mathrm{A}$, the treatment time was $2 \mathrm{~h}$ and relative humidity $60 \%$.

ture of the corona treatment, the conductivity also decreases, for both positive and negative coronas. Although further experiments are under way to understand this temperature effect, one possibility is that higher temperatures promote a greater rate of undesirable recombination reactions, which decreases the number of doping species that reach the sample.

The thermal stability of the electrical conductivity is an important issue to be addressed, especially for producing antistatic polymeric films for electronic packaging. In this context, corona doping appears to be extremely promising. As shown in Fig. 8, positive corona-doped films have much more stable conductivity over the temperature range studied, in comparison to polyaniline samples doped with the conventional $\mathrm{HCl}$ method. This may be attributed to the fact that $\mathrm{HCl}$ is a very volatile dopant for polyaniline, ${ }^{19}$ being eliminated easily when the film is treated above room temperature. For the corona doped PANI on the other hand, the doping species appear either to be less volatile and/or interact more intensively with the PANI backbone.

The effect of corona discharge on the surface morphology of the PANI coating is illustrated in Fig. 9. The globular morphology for untreated PANI (upper image) is modified upon corona treatment (lower image), with the appearance of droplet-like features that are associated with the corona wind in the discharge. Topographical disruption has also been ob-

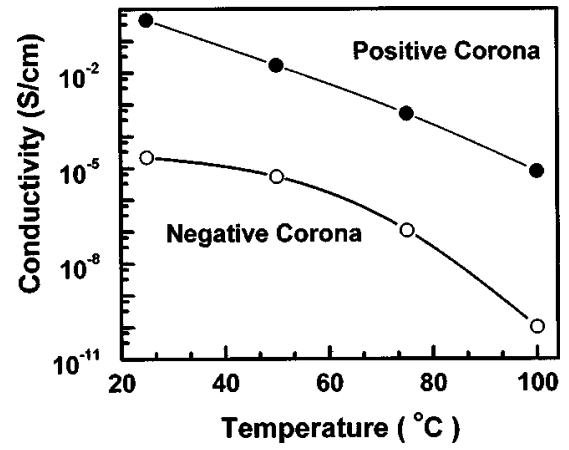

FIG. 7. Dependence of the electrical conductivity for PET/PANI composite films for different treatment temperature. The corona current was $I_{c}$ $= \pm 20 \mu \mathrm{A}, d_{p}=1.3 \mathrm{~cm}$, the treatment time was $2 \mathrm{~h}$, and relative humidity $60 \%$. 


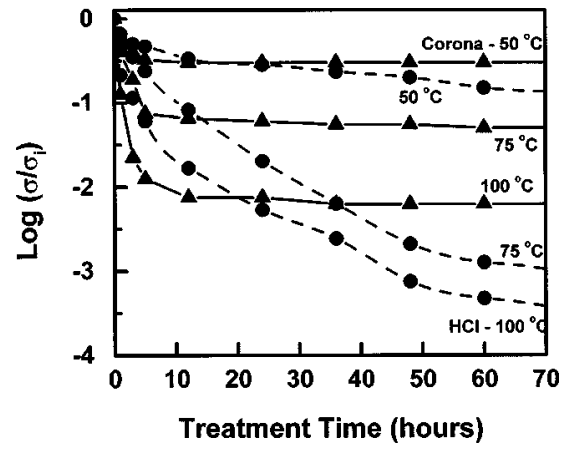

FIG. 8. Decay of the electrical conductivity, $\sigma / \sigma_{i}$ for PET/PANI composites as a function of different temperatures and samples doped by corona discharge and with $\mathrm{HCl}$. Curves were normalized to their initial values, $\sigma_{i}$. Curves marked with triangles and full circles correspond to the results obtained with corona doping and $\mathrm{HCl}$ doping, respectively. Samples were doped at room temperature.

served in the literature for conventional polymers such as corona treated polypropylene films. ${ }^{20}$ From the AFM images, the $\mathrm{Ra}$ and rms roughness values and the average height were calculated. The roughness and height decreased significantly upon corona treatment, with the largest changes being observed up to $60 \mathrm{~min}$ of corona treatment, as reported elsewhere. $^{21}$ One possibility to explain this behavior is that
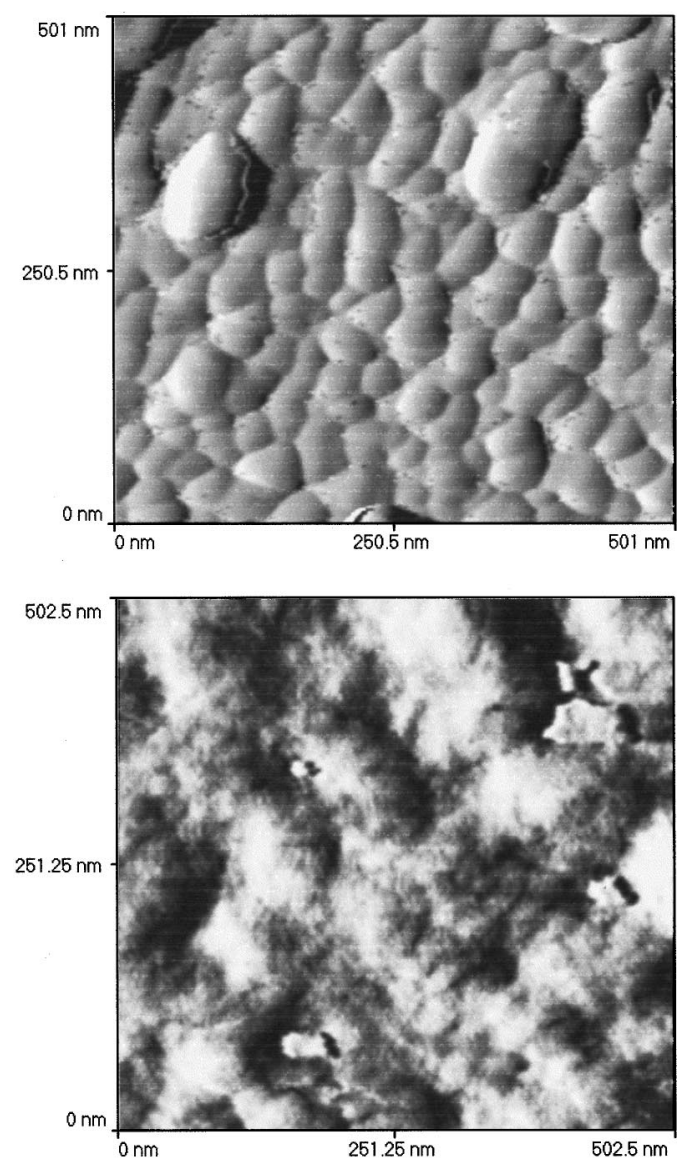

FIG. 9. AFM images of the surface of the PANI layers on PET. Upper image: sample conventionally doped with $\mathrm{HCl}$. Lower image; sample doped using the positive corona discharge. Note that the scales of the pictures are almost the same. the corona wind produced sweeps away the loosely deposited PANI from the top surface leading to a more uniform topography. A similar result was found for the negative corona treatment.

\section{DISCUSSION}

It is well known, that the emeraldine base form of polyaniline is an established example of doping of an organic conjugated polymer into the highly electrically conductive regime as achieved by a protonation process. This is commonly accomplished by treating emeraldine base with aqueous protonic acids, the most widely used being hydrochloric acid at $1.0 \mathrm{M}$ concentration. It has been also shown in the literature that corona discharge produces protonated species, as we will discuss below, which suggests penetration within the polyaniline, promoting the doping observed.

The hypothesis of protonic doping by corona is corroborated by the recent work of Held and Peyrous ${ }^{22}$ and Pinart, ${ }^{23}$ as well as by previous ion/water cluster formation studies. ${ }^{24}$ Corona discharge gives enough energy to produce the dissociation of simple molecules $\mathrm{N}_{2}, \mathrm{O}_{2}$, and $\mathrm{H}_{2} \mathrm{O}$ in humid air. As a result very reactive atoms or radicals such as $\mathrm{N}, \mathrm{O}, \mathrm{H}$, and $\mathrm{OH}$ are formed and can recombine very rapidly either among themselves or with original molecules to give new products which depend strongly on the polarity of the discharge. Using a positive point-to-plane corona discharge in air in the presence of water, hydrated protons were one of the main products detected by Held and Peyrous, ${ }^{22}$ which is supported by previous work indicating the formation of protonated water clusters by corona. ${ }^{24}$ They $^{22}$ also observed for unchanged pressure conditions, similar to those used in this work, that the corresponding ions signal increased with current. This behavior is consistent with our results which demonstrate a conductivity increase as a function of the discharge current. The case of the species generated by the negative corona discharge was studied by Pinart et al. ${ }^{23}$ They suggested that excited species of $\mathrm{NO}_{2}, \mathrm{OH}, \mathrm{N}$, and $\mathrm{O}_{2}$ are subjected to many collisions during their transport towards the plane, leading to the formation of acidic substances i.e., $\mathrm{HNO}_{2}, \mathrm{HNO}_{3}$, on the sample. The presence of dissociated $\mathrm{NO}_{2}^{-}$and $\mathrm{NO}_{3}^{-}$ions in the sample was also observed. The increase in their concentration was accompanied by a linear increase in the proton concentration (slope of 1), due to the acid dissociation in the sample. Such phenomena can thus explain the observation of doping by a protonation process in the case of negative corona, in spite of polarity. In this case they further observed that the concentration of the acid ions described above also increases with the corona current intensity, consistent with our results.

In summary, positive corona discharge produces positively charged and protonic ions that reach the polyaniline surface. By contrast, negative corona lead to the formation of neutral acidic substances which reach the polymer surface and can dissociate within the sample in the presence of moisture leading to protonic species. This hypothesis is further corroborated by the more efficient doping achieved with the positive corona, since the species produced are already in a dissociated protonic form. It seems likely, therefore, that 
both corona discharges can result in the generation of protonic ions, which penetrate within the polyaniline, promoting a protonation effect of the polyaniline backbone similar to that which occurs in conventional solution doping. Preliminary electron paramagnetic resonance (EPR) analysis are consistent with the formation of a polysemiquinone radical cation structure, consisting of two separated polarons due to the protonation of the imine sites of the polyaniline backbone, similar to that which occurs in conventional doping. Further work using EPR, Fourier transform infrared (FTIR), and Raman spectroscopy is being carried out and will be published elsewhere.

In comparison with the conventional doping process employing aqueous acid solutions, the corona method eliminates the use of chemicals and enables the use of a continuous, rapid and dry doping method. If a setup comprising a wire-grid-plane arrangement is used, samples may be treated in the form of a roll in which the composite PET/PANI passes under the treatment zone continuously. Such possibilities are extremely important for technological applications of conducting polymers in microelectronics, including antistatic coatings of microchips, sensors, transistors, and other electronic devices. In a few tests we have found that the edge between the doped and nondoped region is very stable, i.e., no spread of the doping region was observed, which allows for possibility of producing different patterns. Corona doping is also efficient for Langmuir-Blodgett films of PANI, whose results will be reported elsewhere.

In conclusion we have demonstrated that the electrical conductivity of PANI coated onto PET films can be increased from $10^{-10}$ to $0.3 \mathrm{~S} \mathrm{~cm}^{-1}$ by exposure to a corona discharge. The conductivity could also be controlled easily by varying parameters such as time of exposure, corona current, and the geometry of the setup where the corona discharge is performed. The method gives very reproducible results; it is a dry and low cost procedure that may be easily controlled.

\section{ACKNOWLEDGMENTS}

Financial support from CNPq, the PADCT program and FAPESP (Brazil) is gratefully acknowledged. The authors also acknowledge Rhodia-Ster (Brazil) for supplying the PET samples and O. N. Oliveira Jr. and R. C. Garrat for the critical reading of the manuscript.

${ }^{1}$ W. S. Huang, B. D. Humphrey, and A. G. Macdiarmid, J. Chem. Soc., Faraday Trans. 82, 2385 (1986).

${ }^{2}$ S. Roth and W. Grauper, Synth. Met. 55-57, 3623 (1993).

${ }^{3}$ W. S. Huang, M. Angelopoulos, J. R. White, and J. M. Park, Mol. Cryst. Liq. Cryst. 189, 227 (1990).

${ }^{4}$ M. Angelopoulos, J. M. Shaw, W. S. Huang, and R. D. Kaplan, Mol. Cryst. Liq. Cryst. 189, 221 (1990).

${ }^{5}$ J. A. Malmonge and L. H. C. Mattoso, Synth. Met. 84, 779 (1997).

${ }^{6}$ M. Wolszczak, J. Kroh, and M. M. Abdel-Hamid, Radiat. Phys. Chem. 45, 71 (1995).

${ }^{7}$ A. E. Job, J. A. Giacometti, and L. H. C. Mattoso, Appl. Phys. Lett. 72, 3279 (1998)

${ }^{8}$ A. E. Job, J. A. Giacometti, and L. H. C. Mattoso, Mater. Res. Soc. Symp. Proc. 488, 879 (1998).

${ }^{9}$ L. H. C. Mattoso, A. E. Job, and J. A. Giacometti, Pending Patent, INPI PI9705332-5, Brazil, 1997.

${ }^{10}$ J. A. Giacometti and O. N. Oliveira, Jr., IEEE Trans. Electr. Insul. 27, 924 (1992).

${ }^{11}$ A. Goldman and J. Amoroux, Electrical Breakdown and Discharge in Gases, Macroscopic Processes and Discharges, edited by E. E. Kunhardt and L. H. Luessen (Plenum, New York, 1983).

${ }^{12}$ C. J. Dias, J. N. Marat-Mendes, and J. A. Giacometti, J. Phys. D: Appl. Phys. 22, 663 (1989).

${ }^{13}$ R. M. Schaffert, Electrophotographic-Part II (Focal, 1975).

${ }^{14} \mathrm{C}$. D. Hendricks, Electrostatics and its Applications, edited by A. D. Moore (Wiley, New York, 1973), Chap. 4.

${ }^{15}$ L. B. Loeb, Electrical Coronas, Their Basic Mechanisms (University of California Press, Berkeley, 1965).

${ }^{16}$ C. Li and Z. Zong, Synth. Met. 44, 23 (1991).

${ }^{17}$ L. H. C. Mattoso, A. G. MacDiarmid, and A. J. Epstein, Synth. Met. 68, 1 (1994).

${ }^{18}$ A. E. Job, P. S. P. Herrmann, Jr., and L. H. C. Mattoso (unpublished).

${ }^{19}$ Y. Wei and K. F. Hsueh, J. Polym. Sci., Polym. Chem. Ed. 27, 4351 (1989).

${ }^{20}$ O. D. Greenwood, R. D. Boyd, J. Hopkins, and J. P. S. Badyal, J. Adhes. Sci. Technol. 9, 311 (1995)

${ }^{21}$ L. H. C. Mattoso, P. S. P. Herrmann, A. E. Job, and J. A. Giacometti, Proceedings of 1st International Conference on Scanning Probe Microscopy of Polymers, Santa Barbara/USA, August 27-29, 1999, p. 13.

${ }^{22}$ B. Held and R. Peyrous, Czech. J. Phys. 49, 301 (1999).

${ }^{23}$ J. Pinart, M. Smirdec, M. E. Pinart, J. J. Aaron, Z. Benmansour, M. Goldman, and A. Goldman, Atmos. Environ. 30, 129 (1996).

${ }^{24}$ M. M. Sahin, J. Chem. Phys. 45, 2600 (1966). 